Canadian Journal of Bioethics

c S B Canadian Journal of Bioethics

Revue canadienne de bioéthique

\title{
L’archéologie de la mort face aux temps récents : pratiques et questionnements éthiques à partir d'une étude de cas
}

\section{Anne Frédérique Richier}

Volume 2, Number 3, 2019

L'Éthique en Archéologie

Ethics in Archaeology

URI: https://id.erudit.org/iderudit/1066472ar

DOI: https://doi.org/10.7202/1066472ar

See table of contents

Publisher(s)

Programmes de bioéthique, École de santé publique de l'Université de

Montréal

ISSN

2561-4665 (digital)

Explore this journal

Cite this article

Richier, A. (2019). L'archéologie de la mort face aux temps récents : pratiques et questionnements éthiques à partir d'une étude de cas. Canadian Journal of

Bioethics / Revue canadienne de bioéthique, 2(3), 146-148.

https://doi.org/10.7202/1066472ar

\section{Article abstract}

Based on an undocumented excavation of a cemetery in use between 1784 and 1905 in Marseille, this commentary aims to decipher the questions of deontology and ethics raised by the archaeology of recent death.
All Rights Reserved @ Anne Frédérique Richier, 2019

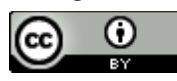

This document is protected by copyright law. Use of the services of Erudit (including reproduction) is subject to its terms and conditions, which can be viewed online.

https://apropos.erudit.org/en/users/policy-on-use/ 


\section{L'archéologie de la mort face aux temps récents : pratiques et questionnements éthiques à partir d'une étude de cas}

\section{Anne Frédérique Richiera,b}

\section{Résumé}

Partant de la fouille inédite d'un cimetière en usage entre 1784 et 1905 à Marseille, ce commentaire vise à décrypter les questions relevant de la déontologie et de l'éthique que pose l'archéologie de la mort récente.

Mots-clés

temps modernes, temps contemporains, devenir des restes humains, translation, conservation, tombe nominative

\section{Abstract}

Based on an undocumented excavation of a cemetery in use between 1784 and 1905 in Marseille, this commentary aims to decipher the questions of deontology and ethics raised by the archaeology of recent death.

\section{Keywords}

modern time, contemporary time, fate of human remains, transfer, preservation, identifiable grave

\section{Introduction}

La fouille, l'étude et la conservation de restes humains issus d'opérations archéologiques posent de façon évidente des questionnements d'ordre déontologique et plus largement éthique. Ces restes ne sont pas comme les autres, même si la démarche scientifique implique une mise à distance avec l'objet d'étude. Ils ne sont pas comme les autres, car ils ne correspondent pas à une «production » humaine, mais à la part, le plus souvent minérale, de ce qui fut un corps, un individu. Dès la fouille, il s'agit de plonger dans l'intimité d'une personne disparue, de dégager et nettoyer ses restes puis d'estimer son âge, son sexe, sa stature, de reconnaître ses maladies, ses carences, son alimentation, ses blessures, ses activités quotidiennes et parfois même son origine, sa religion voire son identité. Cette intimité prend encore plus de relief quand les morts découverts et étudiés sont proches temporellement et que leur contexte d'ensevelissement n'est pas exceptionnel et donc lointain, comme dans le cas des conflits ou des épidémies. À partir de l'exemple d'une fouille préventive menée récemment dans un petit cimetière marseillais en usage entre 1784 et 1905, il s'agit d'aborder, en marge de l'intérêt scientifique et patrimonial du site, les questions déontologiques et éthiques posées par cette opération archéologique et dans certains cas les réponses qui y ont été apportées.

\section{Avant la fouille}

Un diagnostic archéologique mené à l'orée des quartiers nord de Marseille dans le cadre des travaux de prolongement du métro a révélé en 2012 la présence d'un ancien cimetière disparu du paysage et des mémoires. Les recherches en archives ainsi que le mobilier découvert ont permis de reconnaître le cimetière dit « des Crottes » en usage entre 1784 et 1905 . Dans l'attente de la décision des services de l'État de prescrire ou non une fouille archéologique, l'équipe d'archéologues investie dans cette opération a commencé à se poser des questions : peut-on et doit-on fouiller un cimetière de l'époque de nos propres grands ou arrières grands-parents? Est-il opportun d'échantillonner et si oui, selon quels critères et que faire des os humains restants? Y a-t-il des risques sanitaires ou psychologiques pour l'équipe du fait de la proximité temporelle? Que faire dans le cas de sépultures identifiées? Ces questions, curieusement discrètes, voire absentes des discours archéologiques, sont apparues légitimes puisque cette fouille, si elle se faisait, deviendrait la première opération d'archéologie préventive de grande ampleur menée en France sur un cimetière utilisé jusqu'au début du $X X$ e siècle. Les périodes moderne et contemporaine ont en effet été peu investies archéologiquement avant le XXle siècle, malgré un cadre législatif ouvert, sans bornes chronologiques à partir du moment où les sites répondent à des problématiques de recherche et à des enjeux patrimoniaux. La meilleure preuve en est fournie par la date toute récente de leur inscription comme axe dans la programmation nationale archéologique française 1 . Le développement en France de l'archéologie de la Grande Guerre (19141918) depuis les années 1990 a toutefois permis de montrer dans une certaine mesure [1] l'intérêt d'archéologiser les temps récents, même si cela fait encore débat, en France comme en Europe, et plus encore lorsqu'il s'agit de sites funéraires, posant des questions à la fois légales, mémorielles et éthiques [2-4].

Pour revenir au petit cimetière marseillais, le Service Régional Archéologique (SRA) de la région PACA a émis un arrêté pour la réalisation d'une opération de fouille permettant une sauvegarde par l'étude de la totalité de l'emprise concernée par les travaux d'aménagement. Certaines questions susceptibles de se poser à la fouille ont été anticipées dans le cahier des charges, concernant notamment la présence de tombes nominatives. Dans ce cas, une concertation entre l'aménageur, le SRA, l'opérateur de fouilles et la Ville de Marseille, compétente en matière de police funéraire, devrait être engagée afin de statuer sur le sort des restes humains exhumés, avec « en particulier une recherche de descendants dans la perspective d'une restitution à la famille ». L'on peut apprécier ici la spécificité de la fouille de contextes récents, qui peut faire resurgir une dimension mémorielle liée à l'individu (même si celui-ci n'est pas un personnage illustre), rarement rencontrée en archéologie. 


\section{Pendant la fouille}

L'opération de fouille, menée par l'Institut National de Recherches Archéologiques Préventives (Inrap), s'est déroulée en deux tranches d'une durée totale de 10 mois, en 2013 puis en 2014. Elle a permis la découverte de 804 structures funéraires (sépultures en place et ossuaires), des murs d'enceinte du cimetière, plusieurs fois agrandi, et du paysage environnant. Une attention particulière a été portée à l'éventuel impact psychologique d'une telle fouille sur l'équipe : possibilité d'être affecté sur un autre chantier si besoin, réunions et discussions régulières, interventions fréquentes du conseiller sécurité prévention, alternance des tâches... L'équipe, constituée en grande partie de jeunes archéologues expérimentés dans le domaine funéraire2, s'est révélée particulièrement enthousiaste et ouverte à la fouille de contextes récents. Aucune matière organique humaine ou phanères (poils, ongles, cheveux) n'a été rencontrée malgré la présence fréquente d'autres matières organiques habituellement corrompues comme le textile, le cuir ou le bois. Quelques découvertes peu habituelles, voire déroutantes, ont tout de même émaillé les fouilles, comme un enfant inhumé avec une petite carabine, un homme enterré avec une bouteille de vin bon marché contenant un papier malheureusement illisible ou encore un bocal pharmaceutique déposé dans une tombe contenant les restes d'un fœtus... Plusieurs noms inscrits sur des stèles sont sortis de l'oubli, mais sans n'être jamais associés à un ou plusieurs squelettes en place. Ainsi, le problème des tombes nominatives ne s'est pas posé sur cette opération, mais en revanche celui de la destination de restes humains récents est apparu en cours de fouille.

À l'issue de la première campagne de fouilles, il s'est en effet avéré que le nombre de structures funéraires était bien supérieur à celui qui avait été estimé dans le cahier des charges, ne permettant pas de traiter toute l'emprise concernée par la prescription archéologique. Les services de l'État ont donc proposé, en concertation avec l'aménageur et l'opérateur, de fouiller le maximum de structures grâce à un avenant au contrat initial, mais de traiter rapidement un secteur sans conserver les ossements humains présents à l'intérieur, en prévoyant une translation vers le grand cimetière de la ville, « assurant un lieu de sépulture décent et définitif à ces restes humains qui ne feront pas l'objet d'études scientifiques en post-fouille » (addendum au cahier des charges). La translation a concerné une vingtaine de dépôts, qui ont été topographiés et rapidement prélevés par des membres de l'équipe. Cette tâche peu gratifiante a toutefois été confiée aux archéologues les plus aguerris pour ne pas heurter les sensibilités des plus jeunes, pouvant ne pas comprendre les différences de traitement. Les ossements contenus dans chaque structure ont été individualisés dans des sacs numérotés puis ont été remis à des agents funéraires du cimetière Saint-Pierre de Marseille afin qu'ils les placent dans des « reliquaires » (boîtes en bois de taille restreinte) déposés de façon pérenne dans l'ossuaire municipal. L'anticipation de cette opération particulière de translation et son cadrage précis constituent à notre connaissance une première en la matière, aucun cadre législatif ou même déontologique n'existant en France hormis les articles 16-1-13 du Code civil et 225-174 du Code pénal [5].

\section{Après la fouille}

Les résultats scientifiques de cette opération inédite ont montré l'intérêt de fouiller les cimetières récents lorsqu'ils sont menacés, même si de nombreuses autres sources, non intrusives et non destructrices5, sont disponibles [6]. Ils ont en effet permis de documenter l'évolution des pratiques funéraires et de la population durant une période de grandes mutations, révélant souvent une histoire "souterraine », peu ou pas renseignée par ailleurs. L'étude de la gestion des corps et de la constitution d'ossuaires ont par exemple mis en exergue des gestes singuliers de la part des fossoyeurs, entrant souvent en contradiction avec le tout nouveau cadre législatif [7]. De même, l'étude du recrutement des populations exhumées a ouvert des thématiques sociales comme celles des inégalités dans la vie et dans la mort, du poids religieux dans les pratiques funéraires ou des phénomènes migratoires [8]. Ainsi, il a été possible de mettre en regard le cadre normatif révélé par les sources historiques et la réalité des pratiques et des gestes, souvent toute différente. Depuis cette opération, quelques autres fouilles archéologiques de cimetières fonctionnant au XIXe siècle ont été réalisées ou prescrites, mais elles restent marginales puisque la communauté archéologique n'est pas pleinement convaincue de leur intérêt [9]. Cette légitimité scientifique et patrimoniale qui peine à être acquise pose une vraie question de société : les vestiges humains des périodes récentes menacés par les travaux d'aménagement doivent-ils être considérés comme biens culturels bénéficiant du principe d'inaliénabilité et sinon, doivent-ils être réenfouis, voire détruits sans autre forme de procès6, faisant disparaître le support minéral de la mémoire pour les générations futures? Cette question de statut et de conservation apparaît encore plus brûlante à une époque où le problème de place dans les cimetières en activité se résout par la crémation des restes osseux et la reprise d'emplacement de concessions7. Quelle place la société est-elle prête à céder à ses morts plus ou moins anciens à une époque où le rapport à la mort s'intimise de plus en plus, au détriment du collectif [5]? Le récent colloque construit autour de la question «que vont devenir les cimetières en Normandie et ailleurs? »8 qui rassemblait historiens, sociologues, anthropologues, juristes, conservateurs de cimetières et élus a fait date sur ce sujet et offert à l'archéologie l'occasion de s'inscrire dans un débat de société bientôt majeur [4].

\footnotetext{
2 Nombre d'archéologues en poste n'ont toutefois pas souhaité participer à cette fouille funéraire du fait de son caractère récent, certains la qualifiant même de « dégoûtante ».

${ }_{3}$ Article qui rappelle que les restes humains, même crémés, doivent être traités avec « respect, dignité et décence ».

4 Article qui cadre et punit, sans mention temporelle, toute atteinte au respect dû aux morts : violation ou profanation de sépulture, atteinte à l'intégrité du cadavre.

5 Archives historiques, documents graphiques et photographiques, témoignages, ethnographie, littérature, arts...

${ }_{6}$ Comme cela s'est largement pratiqué au XXe siècle pour des sites modernes et contemporains dans le cadre de travaux d'aménagement.

7 Ce qui est le cas même pour les concessions «perpétuelles » en cas d'abandon manifeste (Art. 2223-17 du CGCT).

${ }_{8}$ Colloque organisé par Jacky Brionne, Gaëlle Clavandier et François Michaud-Nérard en août-septembre 2017 à Cerisy-la-Salle (publication à paraître).
} 
Au-delà des questions scientifiques et mémorielles posées par l'archéologie de la mort récente, une dimension éthique, mais surtout déontologique est présente au cœur même du métier d'archéo-anthropologue. Car travailler sur la mort, cette salope, pour reprendre les mots de la thanatologue québécoise Luce des Aulniers, implique bien sûr une posture de recherche dictée par une approche scientifique, mais la proximité temporelle peut mettre à mal la distance nécessaire entre objet et sujet [10]. Nous avons vu en filigrane que la fouille de morts récents pouvait faire naître de nouvelles questions, de nouveaux problèmes et éventuellement exacerber les sensibilités des chercheurs face à leur objet d'étude. En l'absence de charte déontologique ou même simplement de discours par rapport aux choix pratiques à effectuer et à l'aspect plus « sensible " du métier face aux restes humains, chacun bricole un peu des règles dans les limites conceptuelles de sa propre notion du respect.

\section{Remerciements}

A toutes celles et ceux qui, de près ou de loin, ont participé à cette opération archéologique

\section{Conflits d'intérêts}

Aucun à déclarer

\section{Responsabilités des évaluateurs externes}

Les recommandations des évaluateurs externes sont prises en Reviewer evaluations are given serious consideration by the editors considération de façon sérieuse par les éditeurs et les auteurs dans la and authors in the preparation of manuscripts for publication. préparation des manuscrits pour publication. Toutefois, être nommé Nonetheless, being named as a reviewer does not necessarily denote comme évaluateurs n'indique pas nécessairement l'approbation de ce approval of a manuscript; the editors of Canadian Journal of Bioethics manuscrit. Les éditeurs de la Revue canadienne de bioéthique take full responsibility for final acceptance and publication of an article. assument la responsabilité entière de l'acceptation finale et de la publication d'un article.

Édition/Editors: Charles Marsan \& Aliya Affdal

Évaluation/Peer-Review: Alain Dierkens

\section{Affiliations}

a Institut national de recherches archéologiques préventives, Marseille, France ๖ UMR 7268, Aix Marseille Universitél; CNRS, EFS, ADÉS, Marseille, France

\section{Correspondance / Correspondence: Anne Frédérique Richier, anne.richier@inrap.fr}

\section{Reçu/Received: 11 Nov $2018 \quad$ Publié/Published: 27 Nov 2019}

Les éditeurs suivent les recommandations et les procédures décrites The editors follow the recommendations and procedures outlined in dans le Code of Conduct and Best Practice Guidelines for Journal the COPE Code of Conduct and Best Practice Guidelines for Journal Editors de COPE. Plus précisément, ils travaillent pour s'assurer des Editors. Specifically, the editors will work to ensure the highest ethical plus hautes normes éthiques de la publication, y compris l'identification standards of publication, including: the identification and management et la gestion des conflits d'intérêts (pour les éditeurs et pour les of conflicts of interest (for editors and for authors), the fair evaluation auteurs), la juste évaluation des manuscrits et la publication de of manuscripts, and the publication of manuscripts that meet the manuscrits qui répondent aux normes d'excellence de la revue.

\section{Références}

1. Desfossés Y., Jacques A., Prillaux G. L’archéologie de la Grande Guerre. Paris : Editions Ouest-France ; 2008.

2. Tarlow S. The Archaeology of Death in Post-medieval Europe. De Gruyter Open ; 2015.

3. Richier A. Pourquoi fouiller les cimetières du XIXe siècle? L'exemple du site des Crottes à Marseille. Revue d'histoire du XIXe siècle, numéro thématique 2017/2 : Histoire et archéologie : que faire du XIXe siècle? Société de 1848. Paris. A paraître.

4. Richier A. Le cimetière contemporain : un « objet » archéologique? Actes du colloque de Cerisy-la-Salle, Que vont devenir les cimetières en Normandie et ailleurs? Septembre 2017. À paraître.

5. Clavandier G. Inhumer les restes humains anciens. Des enjeux juridiques et éthiques, aux pratiques sur le terrain. Rencontre autour de nos aïeux - La mort de plus en plus proche, Actes de la 8ème rencontre du Groupe d'Anthropologie et d'Archéologie Funéraire, 25-27 mai 2016, Marseille. À paraître.

6. Bertherat $B$, ed. Les sources du funéraire en France à l'époque contemporaine. Editions universitaires d'Avignon ; 2015.

7. Richier A. Au-delà de la sépulture : les ossuaires dans les cimetières modernes et contemporains (XVle-XIXe s.). In : Lauwers et Zemour (eds.). Qu'est-ce qu'une sépulture? Humanités et systèmes funéraires de la Préhistoire à nos jours, XXXVle rencontres internationales d'archéologie et d'histoire d'Antibes. Antibes : Editions APDCA ; 2016:261-277.

8. Richier A, Weydert N. La présence italienne en Provence à partir de la fouille archéologique de cimetières (XVleXXe s.). Diasporas. 2017;30:19-33.

9. Weydert N. et al., eds. Rencontre autour de nos aïeux : la mort de plus en plus proche, Actes de la 8e Rencontre du Groupe d'anthropologie et d'archéologie funéraire, 25-27 mai 2016, Faculté de la Timone, Marseille. Reugny: Editions du Gaaf ; 2019.

10. Bonnabel L, Richier A. Y a-t-il un cadavre dans la tombe? Techniques \& Culture. 2013;60: 74-91. 